\title{
DIAGNOSIS DE ALIMENTOS CON ORIGEN TRANSGÉNICO EN LA FRONTERA URUGUAY- BRASIL: LEGISLACIÓN, CONOCIMIENTO Y ROTULADO
}

\author{
DIAGNOSIS OF FOODS WITH TRANSGENIC ORIGIN ON THE URUGUAY-BRAZIL BORDER: LEGISLATION, \\ KNOWLEDGE AND LABELING.
}

Traversa Tejero Ignacio Pablo*.

Centro Regional de Profesores, Uruguay.

\section{RESUMEN}

Introducción. Los alimentos con ingredientes de origen transgénico están globalizados y el cultivo de semillas transgénicas modifica las matrices productivas de los países del Mercosur. Objetivo: Comparar la legislación de los alimentos con ingredientes transgénicos de Uruguay y Brasil, analizar el rotulado, y evaluar el conocimiento de la población fronteriza de ambos países. Material y Método: Se analizó la evolución del marco jurídico, el relevamiento de alimentos comercializados en supermercados y la aplicación de cuestionarios a los habitantes de dos municipios. Resultados: Muestran similitud de los marcos jurídicos. En Uruguay están autorizados maíz y soja, en Brasil, están, además, frijol, caña de azúcar, algodón y eucalipto. El rotulado es exigido cuando un alimento supera el 1\% de componentes de origen transgénico. Los alimentos transgénicos vendidos en supermercados totalizan 38. Los ingredientes mayoritarios son harina de maíz y aceite de soja transgénicos. El $25 \%$ de la población comprende el proceso biológico para la obtención de un alimento transgénico y el $20 \%$ sabe identificar el rotulado de alimento transgénico. Conclusiones: La autonomía alimentaria se logra con mejor información, discusión de modelos productivos y reflexión sobre sostenibilidad de paradigmas socioeconómicos.

Palabras Clave: Modelo productivo, legislación, rotulado, autonomía alimentaria.

\section{ABSTRACT}

Introduction: Foods with ingredients of transgenic origin are globalized and the cultivation of transgenic seeds modifies the productive matrices of the Mercosur countries. Objective: To compare the legislation of foods with transgenic ingredients in Uruguay and Brazil, analyze the labeling, and evaluate the knowledge of the border population of both countries. Material and method: Consisted of the analysis of the evolution of the legal framework, the survey of foods sold in supermarkets and the application of questionnaires to the inhabitants of two municipalities. Results: Similarity of legal frameworks was. In Uruguay, corn and soybeans are authorized, in Brazil, there are also beans, sugar cane, cotton and eucalyptus. Labeling is required when a food exceeds $1 \%$ of components of transgenic origin. Transgenic foods sold in supermarkets total 38 . The main ingredients are transgenic corn flour and soybean oil. $25 \%$ of the population understand the biological process to obtain a transgenic food and $20 \%$ know how to identify the labeling of transgenic food. Conclusion: Food autonomy is achieved with better information, discussion of production models and reflection on the sustainability of socioeconomic paradigms. Key words: Production model, legislation, labeling, food autonomy.

Correspondencia: Ignacio Pablo Traversa Tejero. igtraversa@gmail.com

Recibido: 13 de abril 2021, aceptado: 29 de julio 2021

(C) Autor2021

\section{(c) (1)}

DOI: https://doi.org/10.29105/respyn20.4-5

Citation: Traversa Tejero I.P. (2021) Diagnosis de alimentos con origen transgénico en la frontera

Uruguay-Brasil: Legislación, conocimiento y rotulado. Revista Salud Pública y Nutrición, 20 (4), 47-56. 


\section{Introducción}

Los cultivos transgénicos aumentan en superficie año tras año desde el inicio de su comercialización en 1996; incluyen importantes fuentes alimentarias, como el maíz, el arroz, el trigo y la soja, esa lista se complementa con algodón, canola y varias hortalizas. En el MERCOSUR (Mercado Común del Sur) Brasil cultiva 25,5 millones de hectáreas (ha) y Argentina 23 millones ha. Por su parte, Paraguay implanta unos tres millones de ha transgénicas, y recientemente Uruguay ha superado el millón de hectáreas con cultivos biotecnológicos (FAO, 2021). Fue a fines de la década de 1990, cuando en el MERCOSUR se introdujeron los primeros cultivos genéticamente modificados (CGM) junto a nuevas tecnologías, insumos y recursos financieros (Terradas, 2017). Las semillas transgénicas darían mayores cosechas y serían una solución al hambre en el mundo, sin embargo, el hambre hallaría su razón en la desigual distribución de la riqueza (RAPAL, 2019). En Uruguay, luego de veinte años, el nuevo modelo productivo introducido no ha cumplido su promesa de disminución de hambre y de uso de agroquímicos (Martínez Debat, 2018). Se espera que la producción de soja en Brasil crezca a razón de 2,6\% anual, al tiempo que en Argentina a una tasa de $2,1 \%$ anual y en Estados Unidos con un incremento de $1,0 \%$. La proyección prevé que las exportaciones de la oleaginosa en 2026 estarán dominadas por Brasil y Estados Unidos, que juntos representan casi el $80 \%$ de las exportaciones mundiales (Gutiérrez, 2011).

Las actuales políticas económicas articulan y reproducen un contexto de dependencia mundial e inserción de los recursos naturales a los flujos del comercio mundial (Segrelles Serrano, 2005), al punto que en Uruguay, la ganadería extensiva y los rubros cerealeros de: maíz, girasol, trigo, cebada, avena y sorgo, comenzaron a ser desplazados por cultivos forestales y cultivos transgénicos de soja y maíz (Achkar et al., 2006) que cambiaron la matriz productiva del país (Arbeletche y Carballo, 2006) y promovieron la investigación de cultivares de uso comercial para la exportación de semillas de soja (Terradas, 2017).

Los países del MERCUSOR han incorporado organismos específicos para la evaluación de los OGM (organismos genéticamente modificados). Las regulaciones pretenden garantizar que los OGM que son liberados al medio sean seguros para el ecosistema. En Brasil, la normativa que rige está presente en la ley de Bioseguridad; en Paraguay y Uruguay, en función de decretos del poder ejecutivo y en la Argentina, por medio de resoluciones y normativas específicas (CAS/IICA, 2010).

De acuerdo con Galeano (2017), en 2017 se encontraban autorizados en Uruguay, los cultivos transgénicos de maíz y de soja, para el primero se autorizaron diez eventos y para el segundo fueron cinco; las empresas autorizadas fueron: Monsanto, Syngenta, Pioneer-Dow, Bayer y BASF.

La inocuidad en los alimentos transgénicos se encuentra cuestionada por la comunidad científica quien identifica efectos adversos a nivel toxicológico y alergénico (Domingo y Bordonaba, 2011). Dentro del periodo 2002-2015, el herbicida más utilizado fue el glifosato, el cual acompañó el crecimiento del área sembrada con soja (Terradas, 2017). La soja genéticamente modificada cultivada es resistente al glifosato, gracias al gen RR (Round-up Ready) introducido en su ADN (Blum et al., 2008).

La liberación de un nuevo evento transgénico está sujeta a un procedimiento de evaluación de riesgo ambiental (Terradas, 2017) y la discusión sobre cultivos transgénicos y alimentos derivados se mantiene en debate en Uruguay; los cultivadores de arroz están en contra de las variedades OMG (García et al., 2010). Para algunos, la introducción de estos cultivos provoca pérdidas de los servicios ecosistémicos, erosión de suelos y transformaciones socioeconómicas (Terradas, 2017) y de acuerdo con Jurkiewicz et al., (2014), la preocupación por los alimentos transgénicos entre los consumidores se mantiene debido a la falta de estudios acerca de los efectos.

La Ingeniería Genética opera por medio de enzimas de restricción; toma un fragmento de ADN y con el auxilio de una enzima ADN ligasa se une a un vector o plasmidio generando una molécula recombinante. El transgénico se obtiene con el ADN recombinante que se introduce en un microorganismo cultivado. $\mathrm{Al}$ desarrollarse expresa el gen de interés en el vegetal (cultivo). De acuerdo con Hilbeck y Schmidt (2006), una gran variedad de estudios comprueba que las toxinas $\mathrm{Bt}$ (Bacillus thurigiensis) presentes en los 
cultivos transgénicos son nocivas para veinte especies de organismos benéficos en los ecosistemas. Pese a que Brasil y Argentina ocupan el segundo y tercer puesto dentro de los países productores de cultivos genéticamente modificados en el mundo, en Latinoamérica hay un bajo nivel de conciencia y conocimientos acerca de la biotecnología (Evans y Ballen, 2013). Por lo expuesto, el presente trabajo plantea la hipótesis de la existencia de comercialización de alimentos con ingredientes de origen transgénico en la región fronteriza entre Uruguay y Brasil y por ello, se hace necesario conocer las perspectivas de los consumidores en relación a los alimentos de origen transgénicos (Bianco et al., 2010). El objetivo de esta investigación fue comparar la legislación de los alimentos con ingredientes transgénicos en Uruguay y Brasil, analizar la presencia del rotulado, y evaluar el conocimiento de la población fronteriza de ambos países.

\section{Material y Método \\ Área de estudio}

La frontera uruguayo brasileña incluye cinco municipios uruguayos y once brasileños. La región presenta una alta concentración urbana centrada en las ciudades capitales (Mazzei y De Souza, 2012). Por razones de representatividad, fueron seleccionados dos municipios, uno uruguayo (Rivera) y otro brasilero (Livramento), ambos localizados en la mitad de la línea fronteriza entre Uruguay y Brasil.

\section{Colecta de datos}

Para el relevamiento de la bibliografía, se realizó un muestreo con el fin de conocer las leyes, decretos y normas que regulan los cultivos y alimentos transgénicos en Uruguay y Brasil, ese relevamiento incluyó portales oficiales de los ministerios de agricultura y medio ambiente de ambos países y otras entidades gubernamentales. Para la selección de los supermercados se aplicó un muestreo aleatorio simple; se escogieron al azar tres mercados de la frontera uruguaya (Rivera) y tres mercados de la frontera brasilera (Livramento). Para determinar los alimentos con ingredientes de origen transgénico, se realizó un censo de cada supermercado, rastreando completamente las góndolas de los mercados y para conocer el origen del ingrediente transgénico se tomaron registros fotográficos de las etiquetas de los alimentos transgénicos (frente $\mathrm{y}$ verso). Como complemento y a los efectos de evaluar el conocimiento de los consumidores, fue aplicado un cuestionario aleatorio in situ, a personas que realizaron compras en los supermercados seleccionados (en total 390 observaciones). El universo de los entrevistados fue definido por los habitantes de las ciudades de Rivera (64.465) y Livramento (76.321), como criterios de inclusión, exclusión y eliminación fueron consideradas solamente personas con mayoría de edad (18 años).

\section{Procesamiento de los datos}

La información colectada referente a normas reguladoras de transgénicos fue analizada organizada cronológicamente y comparada entre ambos países. Los datos de campo provenientes de los supermercados (alimentos transgénicos y entrevistas) fue ingresada por variable de estudio en una planilla de cálculo de Microsoft Excel, en donde fueron aplicadas técnicas de estadística descriptiva numéricas de tendencia central y dispersión, técnicas de tabulación y graficación (Infante y Zárate de Lara, 2015). A los efectos de rechazar o no la hipótesis de trabajo, se tomó como criterio la presencia o ausencia de alimentos con presencia de ingredientes transgénicos en alguno de los seis supermercados analizados.

\section{Resultados y discusión}

Evolución de la legislación uruguaya

El Ministerio de Vivienda, Ordenamiento Territorial y Medio Ambiente, aplica medidas para prevenir y controlar los riesgos ambientales derivados de la biotecnología de creación, manipulación, utilización o liberación de organismos genéticamente modificados en cuanto afecten la conservación y sostenibilidad de la diversidad biológica y el ambiente. Además, coordina medidas a adoptar frente a riesgos con la salud humana, la seguridad industrial y laboral y de buenas prácticas de laboratorio, farmacéutico y alimenticio (Uruguay, 2000).

La introducción, uso y manipulación de vegetales genéticamente modificados, pueden efectuarse previa autorización. Dicha autorización se otorga caso a caso, a partir de los resultados obtenidos en las etapas de la evaluación y gestión del riesgo de una aplicación sobre el ambiente, la diversidad biológica, 
la salud humana, la sanidad animal y vegetal y los aspectos socioeconómicos (Uruguay, 2008).

El Gabinete Nacional de Bioseguridad (GNBio) autoriza nuevas solicitudes vinculadas a los vegetales y sus partes genéticamente modificadas que ingresan al país y modela los lineamientos de políticas nacionales de bioseguridad (Uruguay, 2008), además promovía acciones para la implementación del etiquetado inicialmente voluntario aplicable a aquellos alimentos en los que se comprobaba mediante análisis del producto final la presencia de ADN o proteínas genéticamente modificados (Uruguay, 2008). Ya en 2013, en la ciudad de Montevideo se estableció que los alimentos que eran manipulados genéticamente en más de $1 \%$ del total de componentes, debían ser etiquetados (Montevideo, 2013). En 2018, se dispuso que aquellos alimentos que contenían ingredientes modificados genéticamente, debían llevar en la cara principal del envase el símbolo de transgénicos. Se trata de un símbolo formado por dos círculos concéntricos con una letra $\mathrm{T}$ en el círculo central y con un espacio entre ambos círculos conteniendo la expresión: "contiene organismo modificado genéticamente" (Montevideo, 2018).

Las instituciones públicas y privadas que desarrollan y/o manipulen organismos vegetales genéticamente modificados (OVGM) debe conformar una Comisión Interna de Bioseguridad (CIB) y registrarse ante el GNBio (Uruguay, 2014). La difusión de la información pública correspondiente a la presentación de las solicitudes de estudio de nuevos eventos se realiza mediante la publicación de en la página electrónica del Ministerio de Ganadería Agricultura y Pesca y en diarios de circulación nacional. El GNBio realiza una instancia de instrucción pública con el objetivo de consultar, informar a la ciudadanía y recibir sus pareceres (Uruguay, 2021). Están autorizados los cultivos de soja y de maíz de ciertos eventos (Tabla 1).

\section{Evolución de la legislación brasileña}

En Brasil se establecen las normas para el uso de las técnicas de ingeniería genética y la liberación al medio ambiente de los organismos genéticamente modificados (OGM); la Comisión Técnica Nacional de Bioseguridad (CTNbio) ha elaborado las instrucciones normativas analizando proyectos de experimentación (Brasil, 1995). El rótulo del embalaje o del recipiente en el que está contenidos el alimento debe poseer el nombre del producto transgénico o producto producido a partir de producto transgénico (Brasil, 2003). Esta regulación es reforzada por Brasil (2005) debido a que todos los alimentos e ingredientes alimenticios destinados al consumo humano o animal que contengan o sean producidos a partir de OGM o derivados deben contener información en sus rótulos, conforme reglamento (Brasil, 2005).

\begin{tabular}{|c|c|c|}
\hline Especie/Evento & Uso solicitado & Autorizados \\
\hline Soja 40-3-2 & Comercial & 02/10/1996 (Decreto 249/000 \\
\hline Soja A2704-12 (LL) & Comercial & $19 / 09 / 2012$ \\
\hline Soja A5547-127 (LL) & Comercial & 19/09/2012 \\
\hline Soja MON89788XMON87701 (RR2YBt) & Comercial & 19/09/2012 \\
\hline Soja BPS-CV127-9 & Comercial & $29 / 10 / 2014$ \\
\hline Soja DAS44406-6 & Comercial & En evaluación \\
\hline Soja MON89788XMON87708 & Comercial & En evaluación \\
\hline Soja FG72 & Comercial & En evaluación \\
\hline Soja FG72XA5547-127 & Comercial & En evaluación \\
\hline Maíz MON810 & Comercial & $\begin{array}{l}\text { 20/06/03 (Decreto 249/000) } \\
\text { 21/06/11 (Decreto 353/008) }\end{array}$ \\
\hline Maíz BT11 & Comercial & $\begin{array}{l}\text { 05/05/04 (Decreto 249/000) } \\
\text { 21/06/11 (Decreto 353/008) }\end{array}$ \\
\hline Maíz GA21 & Comercial & $21 / 06 / 2011$ \\
\hline Maíz GA21XBT11 & Comercial & $21 / 06 / 2011$ \\
\hline Maíz TC1507 & Comercial & $21 / 06 / 11,20 / 10 / 11$ \\
\hline Maíz NK603 & Comercial & $21 / 06 / 2011$ \\
\hline Maíz MON810XNK603 & Comercial & $21 / 06 / 2011$ \\
\hline Maíz TC1507XNK603 & Comercial & 19/09/2011 \\
\hline Maíz BT11XMIR162XGA21 & Comercial & 19/09/2012 \\
\hline Maíz MON89034XTC1507XNK603 & Comercial & 19/09/2012 \\
\hline Maíz dulce & Comercial & 19/09/2012 \\
\hline Maíz MON89034XMON88017 & Comercial & En evaluación \\
\hline Maíz BT11XMIR162XMIR604XGA21 & Comercial & En evaluación \\
\hline Maíz MON89034XNK603XTC1507XDAS40278-9 & Comercial & En evaluación \\
\hline Maíz TC1507XMON810XNK603 & Comercial & En evaluación \\
\hline Maíz T25 & Comercial & En evaluación \\
\hline Soja MON89788 (RR2Y) & Semilla/Export. & $\begin{array}{l}\text { 03/11/09, (17/11/10, } \\
\text { 20/10/11, 03/10/12, }\end{array}$ \\
\hline Soja A2704-12 (LL) & Semilla/Export. & 03/11/09,(17/11/10, \\
\hline Soja MON89788XMON87701 (RR2YBt) & Semilla/Export. & $17 / 11 / 10,(20 / 10 / 11)$ \\
\hline Soja A5547-127 & Semilla/Export. & $20 / 10 / 2011$ \\
\hline Soja MON89788X87708 & emilla/Export. & $\begin{array}{l}21 / 09 / 2012 \\
13 / 08 / 14)\end{array}$ \\
\hline Soja DAS44406-6 & Semilla/Export. & $01 / 09 / 2014$ \\
\hline
\end{tabular}

Fuente: Adaptado de ${ }^{[25]}$ (URUGUAY, 2019). MGAP (Uruguay).

La creación de la CTNBio se vincula al establecimiento del Sistema de Información de Bioseguridad, el cual corresponde al ámbito del Ministerio de Ciencia y Tecnología y se encarga del registro de información relacionado con las actividades de análisis, autorización, registro, monitoreo y acompañamiento de los distintos procesos de los OMG y derivados [28] (Brasil, 2005). Además, la CTNBio brinda apoyo y asesoramiento al Gobierno Federal en la formulación, actualización e implementación de OGM y en la evaluación de los riesgos que estos 
alimentos provocan a la salud humana y al medio ambiente (Brasil, 2005).

Desde el año 2015, los alimentos que contienen menos de $1 \%$ de transgénicos, pasaron a expresarse como alimento libre de transgénicos, manteniendo el rótulo solo aquellos que contienen más de este porcentaje (Ottajano, 2015). Esta norma confrontó con la Ley de Bioseguridad (11.105-2005), donde se especificaba en el artículo 40 que cualquier alimento poseedor de transgénicos debía estar rotulado (Brasil, 2005). El artículo 10 de la ley 11.460 del año 2007, establece que están impedidas las investigaciones y el cultivo de organismos genéticamente modificados en tierras indígenas y áreas de unidades de conservación, excepto en Áreas de Protección Ambiental (Brasil, 2007).

Por otro lado, las evaluaciones del riesgo a la salud humana y animal precisan del análisis comparativo de la composición química y nutricional entre el alimento oriundo del OGM y el no modificado, in natura, o después del procesamiento y la existencia de equivalencia sustancial entre el OGM y su organismo parental, incluyendo el análisis de antinutrientes, si hubiere (Brasil, 2020).

Están autorizados los cultivos de: soja, maíz, poroto, caña de azúcar, algodón, eucalipto (Brasil, 2003) (Ver tabla 2).

\section{Rotulado de alimentos.}

El rotulado tiene tres propósitos principales, en primer lugar, suministrar información sobre salud y seguridad, en segundo término, proteger consumidores de industrias de embalaje fraudulentas e ilusorias y por último promover competencia leal y marketing de productos (Pozetti, 2014). Los derechos de los consumidores están consagrados en los marcos jurídicos que garantizan la calidad nutricional y la inocuidad sanitaria y la información sobre los atributos intrínsecos de los productos a adquirir para su consumo (Pessanha, 1998). En los supermercados fronterizos de ambos países se alcanzó un total de 38 productos (alimentos) con ingredientes de origen transgénico en su composición, por lo que no se rechaza la hipótesis de la investigación planteada en la introducción referente a la existencia de alimentos "transgénicos en el mercado".

\begin{tabular}{|c|c|c|}
\hline OGM (especie/evento) & Uso solicitado & Autorizado* \\
\hline Soja (Glycine max) & Tolerante Herbicida & $1998 / 1$ \\
\hline Soja (Glycine max) & Tolerante Herbicida & $2009 / 1$ \\
\hline Soja (Glycine max) & Tolerante Herbicida & $2010 / 2$ \\
\hline Soja (Glycine max) & Tolerante Herbicida/Resistencia insectos & $2010 / 1$ \\
\hline Soja (Glycine max) & Tolerante Herbicida & $2015 / 4$ \\
\hline Soja (Glycine max) & Tolerante Herbicida/Resistencia insectos & $2016 / 1$ \\
\hline Soja (Glycine max) & Tolerante Herbicida & $2016 / 1$ \\
\hline Soja (Glycine max) & Tolerante Herbicida & $2017 / 1$ \\
\hline Soja (Glycine max) & Resistencia insectos & $2017 / 1$ \\
\hline Soja (Glycine max) & Tolerante Herbicida/Resistencia insectos & $2018 / 1$ \\
\hline Maíz (Zea mays) & Tolerante Herbicida & $2007 / 1$ \\
\hline Maíz (Zea mays) & Tolerante Herbicida/Resistencia insectos & $2007 / 1$ \\
\hline Maíz (Zea mays) & Resistencia insectos & $2007 / 1$ \\
\hline Maíz (Zea mays) & Tolerante Herbicida & $2008 / 2$ \\
\hline Maíz (Zea mays) & Tolerante Herbicida/Resistencia insectos & $2008 / 1$ \\
\hline Maíz (Zea mays) & Tolerante Herbicida & $2009 / 1$ \\
\hline Maíz (Zea mays) & Tolerante Herbicida/Resistencia insectos & $2009 / 3$ \\
\hline Maíz (Zea mays) & Resistencia insectos & $2009 / 1$ \\
\hline Maíz (Zea mays) & Tolerante Herbicida/Resistencia insectos & $2010 / 3$ \\
\hline Maíz (Zea mays) & Resistencia insectos & $2010 / 1$ \\
\hline Maíz (Zea mays) & Tolerante Herbicida/Resistencia insectos & $2011 / 3$ \\
\hline Maíz (Zea mays) & Tolerante Herbicida/Resistencia insectos & $2013 / 1$ \\
\hline Maíz (Zea mays) & Tolerante Herbicida/Resistencia insectos & $2014 / 2$ \\
\hline Maíz (Zea mays) & Tolerante Herbicida/Resistencia insectos & $2015 / 13$ \\
\hline Maíz (Zea mays) & Tolerante Herbicida/Resistencia insectos & $2016 / 6$ \\
\hline Maíz (Zea mays) & Resistente a insectos & $2017 / 2$ \\
\hline Maíz (Zea mays) & Tolerante Herbicida/Resistencia insectos & $2017 / 2$ \\
\hline Maíz (Zea mays) & Tolerante a herbicida & $2018 / 2$ \\
\hline Algodón (Gossypium sp.) & Tolerante Herbicida/Resistencia insectos & $2005 / 1$ \\
\hline Algodón (Gossypium sp.) & Tolerante Herbicida/Resistencia insectos & $2008 / 2$ \\
\hline Algodón (Gossypium sp.) & Tolerante Herbicida/Resistencia insectos & $2009 / 2$ \\
\hline Algodón (Gossypium sp.) & Resistencia a insectos & $2009 / 1$ \\
\hline Algodón (Gossypium sp.) & Tolerancia a herbicidas & $2010 / 1$ \\
\hline Algodón (Gossypium sp.) & Tolerante Herbicida/Resistencia insectos & $2011 / 1$ \\
\hline Algodón (Gossypium sp.) & Resistencia a insectos & $2011 / 1$ \\
\hline Algodón (Gossypium sp.) & Tolerante Herbicida/Resistencia insectos & $2012 / 2$ \\
\hline Algodón (Gossypium sp.) & $\begin{array}{l}\text { Restauración de fertilidad (producción de } \\
\text { semillas) }\end{array}$ & $2012 / 1$ \\
\hline Algodón (Gossypium sp.) & Tolerante Herbicida/Resistencia insectos & $2016 / 1$ \\
\hline Algodón (Gossypium sp.) & Tolerante Herbicida/Resistencia insectos & $2017 / 1$ \\
\hline Algodón (Gossypium sp.) & Tolerancia a herbicidas & $2017 / 1$ \\
\hline Algodón (Gossypium sp.) & Aumento de termoestabilidad de amilasa & $2018 / 1$ \\
\hline Algodón (Gossypium sp.) & Disminución del estrés hídrico en la sequía & $2018 / 1$ \\
\hline Algodón (Gossypium sp.) & Tolerante Herbicida/Resistencia insectos & $2018 / 3$ \\
\hline Frijol (Phaseolus vulgaris) & Resistente al Virus del mosaico dorado del frijol & 2011/1 \\
\hline Eucalipto (Eucalyptus sp.) & Aumento volumétrico de madera & 2015/1 \\
\hline $\begin{array}{l}\text { Caña azúcar (Saccharum } \\
\text { officinarum) }\end{array}$ & Resistencia insectos & $2017 / 1$ \\
\hline $\begin{array}{l}\text { Caña azúcar (Saccharum } \\
\text { officinarum) }\end{array}$ & Resistencia insectos & $2018 / 1$ \\
\hline
\end{tabular}

Es posible observar que, dentro de los diez primeros en orden de compra por frecuencias absolutas y relativas, se encuentran los alimentos derivados del maíz transgénico, un cultivo cuya data de autorización como transgénica data desde 2003 en Uruguay y de 2007 en Brasil (tablas 1 y 2 respectivamente). También los derivados de la soja la cual estaba autorizada desde 2003 en Uruguay y 2008 en Brasil. En el presente los alimentos comercializados en supermercados derivados de aquellas autorizaciones son: maíz en grano, harina de maíz, almidón de maíz, aceite de maíz (Ver tabla 3).

En supermercados de Belo Horizonte Mansur et al. (2017) analizan 35 rótulos de veinte marcas de aceites derivados de maíz, canola, girasol y soja; 
encontrando que se respeta el rotulado obligatorio, no obstante, con información complementaria incompleta, por lo que los órganos responsables deberían estandarizar las etiquetas de los alimentos.

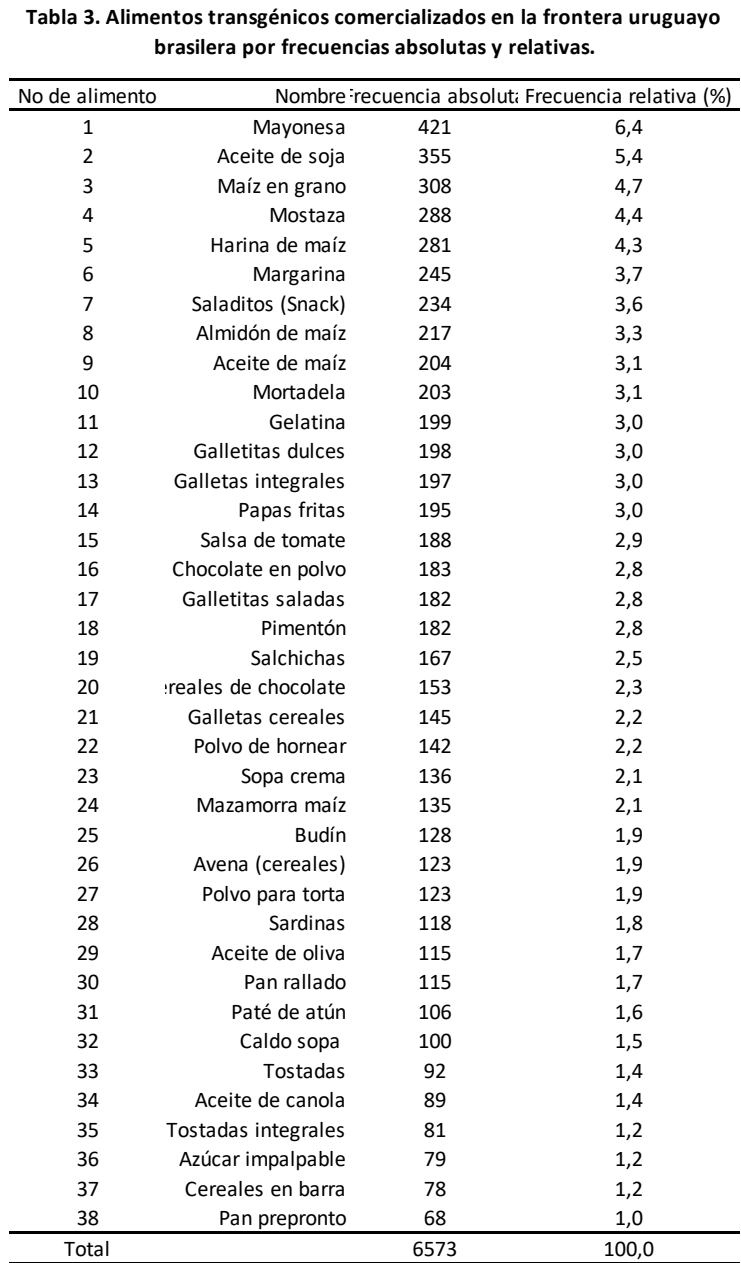

Fuente: el autor.

En una investigación practicada en Trujillo (Perú), se llegó a la conclusión que la población tuvo un bajo nivel de conocimiento sobre los alimentos transgénicos y un alto nivel de consumo de alimentos contemplados como transgénicos, no obstante, un bajo nivel de aceptación de alimentos transgénicos (Rodríguez Soto et al., 2017). En este estudio, casi las tres cuartas partes de la población desconoce la simbología de los alimentos transgénicos, uno de cada cinco los reconoce por la presencia de la letra $\mathrm{T}$.
Esta letra está bajo triángulo equilátero de fondo blanco o amarillo es el símbolo definido por la ordenanza 2658 (Brasil, 2003), apenas el cuatro por ciento reconoce el rotulado en toda la dimensión legal, y el dos por ciento restante identifica el triángulo amarillo de este tipo de alimentos (figura $1)$.

Figura 1. Conocimiento sobre el rotulado de los alimentos transgénicos por frecuencia relativa en porcentaje.

frecuencia r elativa $/ \%$ )

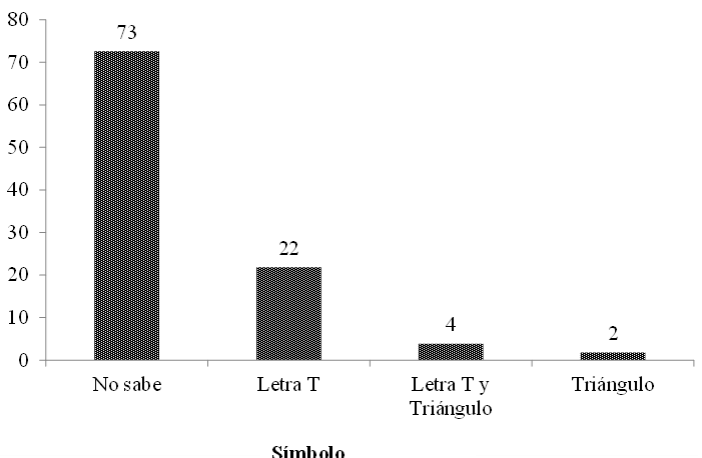

Fuente: el autor

Tomando en consideración el conocimiento que la población tiene sobre la definición de alimento transgénico es posible observar que apenas la cuarta parte registra una idea aproximada, dado que su respuesta fue: "genéticamente modificado, variación de genes o semillas modificadas". El 75\% restante no tiene idea clara sobre lo que consume al comprar un alimento rotulado como de origen transgénico (Ver tabla 4). De acuerdo a Pereira Cavalcante (2020), serían necesarias políticas de estímulo a la educación nutricional de las poblaciones a través de los medios de comunicación. De esta forma se entiende que el rotulado es un paliativo para el consumidor en el mercado de alimentos, porque de acuerdo a HoltGimenez (2009) la importancia radicaría en la soberanía alimentaria entendida esta como antagónica a la promovida por la dominante agricultura de revolución verde (capitalista), la cual está regida por la mercantilización global de los agronegocios. 
Tabla 4. Conocimiento poblacional en la frontera uruguayo brasilera sobre la definición de alimentos transgénicos por frecuencias absolutas y relativas.

\begin{tabular}{rcc}
\hline Conocimiento & Frecuencia absoluta & Frecuencia relativa (\%) \\
\hline no sabe & 139 & 35,3 \\
genéticamente modificado & 83 & 21,1 \\
modificado & 66 & 16,8 \\
artificial & 36 & 9,1 \\
creado en laboratorio & 15 & 3,8 \\
contiene químicos & 11 & 2,8 \\
variación de genes & 15 & 3,8 \\
mejor calidad & 7 & 1,8 \\
con agrotóxicos & 4 & 1,0 \\
perjudicial & 4 & 1,0 \\
más sabroso & 3 & 0,8 \\
no contiene agrotóxicos & 3 & 0,8 \\
hibridación de plantas & 2 & 0,5 \\
mezcla de alimentos & 2 & 0,5 \\
mutación genética & 2 & 0,5 \\
semillas modificadas & 2 & 0,5 \\
\hline Total & 394 & 100,0 \\
\hline
\end{tabular}

Fuente: el autor.

Apenas el 1\% de los respondientes entiende que estos alimentos son diferentes; las respuestas no son coincidentes con un estudio practicado en el estado de Ceará (Brasil), donde el $69 \%$ de la población sostiene que los alimentos con ingredientes transgénicos son como mínimo no saludables o incluso perjudiciales para la salud (Pereira Cavalcante, 2020). Según Ramón Vidal (2018), recién estamos empezando a entender el papel de los transgénicos en la salud y en la enfermedad. Con ello se abren posibilidades de intervención nutricional con probióticos y prebióticos que marcarán en buena medida el futuro de la alimentación y la salud.

\section{Conclusiones}

i) Las leyes que regulan los cultivos y alimentos transgénicos en Brasil se remontan a 1995 y en Uruguay a normas del año 2000. Las legislaciones se enmarcan en la protección del ambiente y el cuidado de la salud y la calidad de los alimentos comercializados. Las autorizaciones de cultivos transgénicos se otorgan caso a caso por órganos competentes; el Gabinete Nacional de Bioseguridad en Brasil, y la Comisión Técnica Nacional de Bioseguridad en Uruguay. Una diferencia entre ambos países consiste en la imposibilidad de la experimentación con cultivos transgénicos, en unidades de conservación y en las tierras indígenas del territorio brasilero. ii) El marco jurídico es muy dinámico y está en consonancia con el surgimiento permanente de las nuevas tecnologías transgénicas del campo agronómico, las cuales obligan a una continua revisión y actualización de las normas ambientales y alimentarias. En Uruguay están autorizados solamente los cultivos transgénicos de maíz y soja, en tanto en Brasil; dada la amplitud geográfica y la diversidad de climas están autorizados, además, frijol, caña de azúcar, algodón y eucalipto.

iii) Es exigido el rotulado cuando un alimento supera el $1 \%$ de componentes de origen transgénico. Los alimentos transgénicos vendidos totalizan 38. El ingrediente mayoritario es derivado de la harina de maíz transgénico y del aceite de soja transgénica. Esos componentes formulan alimentos ampliamente consumidos como: galletitas, papas fritas, cereales de chocolate y polvo de hornear entre otros. Apenas uno de cada cuatro habitantes comprende de forma correcta el proceso biológico necesario para la obtención de un alimento transgénico y uno de cada cinco sabe identificar por la simbología legal (rotulado) el alimento transgénico comercializado en los supermercados.

iv) A los efectos de generar políticas para la protección de la ciudadanía y el fomento de la educación alimentar y nutricional, se hace necesario debatir la temática de la inserción internacional en los modelos productivos de los países y reflexionar sobre la sostenibilidad global de los paradigmas socioeconómicos que los promueven. De esta manera, los habitantes serán portadores de un conocimiento profundo y estarán en la libertad de escoger aquellos alimentos que entiendan saludables dentro de un marco de autonomía alimentaria.

\section{Agradecimientos}

A los pobladores de la faja fronteriza UruguayBrasil, que se prestaron como respondientes de este estudio. 


\section{Bibliografía}

Achkar, M., Domínguez, A., Pesce, F. (2006). Principales transformaciones territoriales en el Uruguay rural contemporáneo. Pampa. Santa Fe, Argentina, UNL. Revista Interuniversitaria de Estudios Territoriales. 2:219-242.

Arbeletche, P., y Carballo, C. (2006). Sojización y concentración de la agricultura uruguaya. En; Libro de resúmenes XXXVII Reunión Anual de la Asociación Argentina de Economía Agrícola. Córdoba, Argentina: AAEA.

Bianco-Bozzo, M., Chiappe-Hernández, M., Carámbula-Pareja, M. (2010). Agrobiotecnologías en Uruguay: posicionamiento de actores en torno a un debate incierto. Agricultura, Sociedad y Desarrollo. 7(3), 247-264. Disponible en: http://www.scielo.org.mx/scielo.php?script=sci arttext\&pid=S1870-

$54722010000300004 \& \operatorname{lng}=\mathrm{es} \& \operatorname{lng}=\mathrm{es}$

Blum, A., Narbondo, I., Oyhantcabal, G., Sancho, D. (2008). Soja transgénica y sus impactos en Uruguay. La nueva colonización. Montevideo: RAP-AL.

Brasil. (1995). Ley 8974/95. Normas para o uso das técnicas de Engenharia genética e liberação no meio ambiente de organismos genéticamente modificados. Disponible en: https://presrepublica.jusbrasil.com.br/legislacao/ 104372/lei-8974-95

Brasil. (2003). Decreto $\mathrm{N}^{\circ} 4680 / 2003$. Direito à informação alimentos e ingredientes alimentares destinados ao consumo que contenham ou sejam produzidos a partir de organismos geneticamente modificados. Disponible en: https://www.saude.rj.gov.br/comum/code/Mostr arArquivo.php?C=MjI0MA $\% 2 \mathrm{C} \% 2 \mathrm{C}$

Brasil. (2003). Portaria 2658/2003. Símbolo transgênico. Ministério da Agricultura, Pecuária e Abastecimento. Ministério da justiça.

Brasil. (2005). Lei $\mathrm{N}^{\circ}$ 11.105. Alimentos transgénicos. Normas de Segurança $e$ mecanismos fiscalização de organismos geneticamente modificados - OGM e derivados.
Artigo 40. Disponible en: http://extwprlegs1.fao.org/docs/pdf/bra51914S.p df

Brasil. (2007). Lei $\mathrm{n}^{\circ}$ 11.460. Plantio de organismos geneticamente modificados em unidades de conservação [citado en 2021 feb 26] Disponible en:

http://www.planalto.gov.br/ccivil_03/_Ato20072010/2007/Lei/L11460.htm

Brasil. (2019). Informacões sobre refúgio solicitadas pela instrução. Tabela de Plantas aprovadas para comercialização Set.pdf. Disponible en: https://www.gov.br/agricultura/ptbr/assuntos/sanidade-animal-e-vegetal/sanidadevegetal/arquivos/TabeladePlantasAprovadaspara ComercializaoSet2019_Refgio.pdf

Brasil. (2020). Resolução Normativa $N^{o} 24$, de 07 de janeiro Comissão Técnica Nacional de Ministério da Ciência, Tecnologia, Inovações e Comunicações.

CAS/IICA. (2010). Marcos regulatorios de bioseguridad y situación de las aprobaciones comerciales de organismos genéticamente modificados en los países del Consejo Agropecuario del Sur. CAS, $2^{\circ}$ edición (on line). Santiago Chile. Disponible en: http://repositorio.iica.int/bitstream/11324/6457/1 /BVE18029646e.pdf

Domingo, JL., Bordonaba, JG. (2011). A literature review on the safety assessment of genetically modified plants. Environment International. 37:734-742.

Evans, E., Ballen, FA. (2013). Synopsis of US Consumer perception of Genetically Modified (Biotech) Crops. Extension Data Information Spurce (EDIS) Insitute of Food and Agricultural Sciences. University of Florida. 5 p. Disponible en:

http://www.akleg.gov/basis/get_documents.asp? session $=30 \&$ docid $=13659$

FAO. (2021). Organización de las Naciones Unidas para la Alimentación y la Agricultura. Actualidad agropecuaria de América Latina y el Caribe. Agronoticias: Actualidad agropecuaria de 
América Latina y el Caribe. Disponible en: http://www.fao.org/in-

action/agronoticias/detail/es/c/506917/

Galeano, P. (2017). Los cultivos transgénicos en Uruguay y el mundo. En: Org. REDES. Amigos de la Tierra Uruguay. Veinte años de cultivos transgénicos en Uruguay. (1). 5-33. Disponible en: https://www.redes.org.uy/wpcontent/uploads/2017/12/Publicacion_20_anios_ de_cultivos_transg\%C3\%A9nicos_en_Uruguay. pdf.

García F, Lanfranco B, Hareau G. (2010). Transgénicos en el cultivo de arroz: implicancias económicas de su adopción en Uruguay. Rev. Agrociencia Uruguay.14 (2):77-88.

Gutiérrez, E. (2017). Transgénicos: La soja gana terreno en toda Sudamérica. Instituto para el desarrollo rural de Sudamérica. IPDRS. Disponible en: https://www.sudamericarural.org/index.php/noti cias/que-pasa/5487-transgenicos-la-soya-ganaterreno-en-toda-sudamerica

Hilbeck, A, Schmidt, JEU (2006). Another View on Bt Proteins- How Specific are They and What Else Might They Do? Biopestic. Int. 2:1-50.

Holt-Giménez, Eric (2009). "From Food Crisis to Food Sovereignty". Montly Review. No.61, pp. 142-56.

Infante Gil, S., Zárate de Lara, G. (2015). Métodos Estadísticos: un enfoque interdisciplinario. México DF: Mundi-Prensa Libros S.A.

Jurkiewicz, A., Zagórski, J., Bujak, F., Lachowski, S., Florek Luszczki, M. (2014) Emotional attitudes of young people completing secondary schools towards genetic modification of organisms (GMO) and genetically modified foods (GMF). Ann Agric Environ Med. 21(1):205-211.

Mansur Rabelo, D., Oliveira Henriques, B., Labanca, RA. (2017). Evaluation of the labeling of vegetable oils according to law: mandatory and optional items. ISSN 2236-6695 Revista a
Barriguda, Campina Grande 7 [1]| P. 023-0034| Jan-Abr.

Martínez Debat, C. (2018). Alimentos transgénicos en Uruguay. UDELAR. Universidad de la República. Facultad de Ciencias. Montevideo, Uruguay. Disponible en: http://www.universidad.edu.uy/prensa/renderIte $\mathrm{m} / \mathrm{itemId} / 42998$

Mazzei, E., De Souza, M. (2012). La Frontera en Cifras. Universidad de la República, Centro de estudios de Frontera. Comisión Coordinadora Interior (CCI). Cerro Largo, Uruguay: CBA.

Montevideo. (2013). Decreto No 34.901. Alimentos que provienen de organismos genéticamente modificados. Junta Departamental de la Intendencia Municipal. Disponible en: https:/imnube.montevideo.gub.uy/share/s/Fphb DiqeSMyPwb5CXp8cSA

Montevideo. (2013). Resolución N N ${ }^{\circ} 22 / 18$. Etiquetado de alimentos provenientes de organismos genéticamente modificados. Junta Departamental de la Intendencia Municipal. [citado en 2021 feb 26] Disponible en: https://montevideo.gub.uy/sites/default/files/bibl ioteca/722-181.pdf

Ottajano, F. (2015). Alimentos Transgénicos. Disponible en: https://ottajano.jusbrasil.com.br/artigos/2499546 42/alimentos-transgénicos

Pereira Cavalcante, JL. (2020). Knowledge and understanding of genetically modified foods by consumers in supermarkets in Tianguá, Ceará, Brazil. SCMS / UNINTA. - UNINTA.SOBRAL, año 9, v.1, n. 16, jan - jun. p. 16 -29.

Pessanha, L. D. R. (1998). Segurança alimentar como um princípio orientador de políticas públicas: implicações e conexões para o caso brasileiro. Tese Doutorado. Universidade Federal Rural do Rio de Janeiro, Rio de Janeiro.

Pozzetti, V. C. (2014). Alimentos transgênicos e o direito do consumidor à informação. Revista Jurídica, $\quad$ 3, n.36. doi: 
http://dx.doi.org/10.21902/revistajur.2316753X.v3i36.993

Ramón Vidal, Daniel. (2018). Biotecnología de alimentos: de los transgénicos a la nutrición personalizada. Nutrición Hospitalaria, 35(spe4), 28-32. Epub 28 de septiembre de 2020.https://dx.doi.org/10.20960/nh.2121

RAP-AL, Uruguay (2006). Red de Acción en Plaguicidas y sus alternativas para América Latina. Transgénicos en Uruguay ¿Ganancia para quién? [Libro electrónico]. Disponible en:http://www.rapaluruguay.org/transgenicos/Ur uguay/librillo/libro.html\#indice

Rodríguez Soto, JC., Contreras Quiñones, M., Espinoza Amaya, CB, Miranda Gallac, ES. (2017). Level of knowledge, consumption and acceptance of transgenic food in settlements of the sector San Andrés, Trujillo 2016. In crescendo, 2017; 8(2): 291-305

Segrelles Serrano, J.A. (2005). El problema de los cultivos transgénicos en américa latina: una "nueva." Entorno Geográfico. 3:93-120.

Terradas, L. (2017). UE versus MERCOSUR: estudio comparados de externalidades ambientales en cultivos transgénicos. Indicadores para Uruguay. [Tesis de Doctorado]. León. Universidad de León. 382 p.

Uruguay. (2000). Ley $\mathrm{n}^{\circ}$ 17.283. Protección del Medio Ambiente. Artículo 23. Bioseguridad. Disponible en:http://euroclimaplus.org/intranet/_documento s/repositorio/Ley\%2017283\%20protecci\%C3\%B3n\%20del\%20Medio\%20Ambi ente_2000Uruguay.pdf

Uruguay. (2008). Decreto $N^{\circ} 353 / 008$ y 535/008. Bioseguridad vegetales genéticamente modificados. Artículo 1 y 4. Disponible en: https://www.impo.com.uy/bases/decretos/3532008

Uruguay. (2014). Resolución GNBio nº5. Procedimiento de autorización para la introducción, uso y manipulación de vegetales y sus partes genéticamente modificados con fines de investigación y/o docencia. Disponible en: file://C:/Documents\%20and\%20Settings/User/ Mis\%20documentos/Descargas/PROCEDIMIE $\mathrm{NTO}+\mathrm{DE}+\mathrm{AUTORIZACION+OVGM+laborato}$ rio+e+invern $\% 2525 \mathrm{c} 3 \% 2525 \mathrm{a} 1$ culo+MARZO2 015.pdf

Uruguay. (2021). Comunicación y Participación Ciudadana. Consulta pública maíz, soja y algodón. Disponible en:https://www.gub.uy/comunicacion/noticias/se -abre-una-nueva-consulta-publica-sobrevegetales-derivados-de-la 